\title{
Hybrid fruit fly optimization algorithm for solving multi-compartment vehicle routing problem in intelligent logistics
}

\author{
Wang, C.L. , Li, S.W. ${ }^{a, *}$ \\ ${ }^{a}$ School of Business, Shandong Normal University, Ji'nan, P.R. China
}

\begin{abstract}
A B S T R A C T
The purpose of this study was to tackle multi-compartment vehicle routing problem in intelligent logistics with the fruit fly optimization algorithm (FOA). A hybrid FOA (HFOA) integrated with three local search methods (2-opt, swap and insert) was adopted to solve the multi-compartment vehicle routing problem (MCVRP) in intelligent logistics by applying discrete space optimization problems. The numerical experiments show that the HFOA algorithm has improved the performance for all proposed problems, including improving the total path length and enhancing the solution quality. The improvement rate in total path length shifts from $3.21 \%$ at 50 customers to $9.83 \%$ at 150 customers indicating that this HFOA is more effective in largescale. The HFOA integrated with 2-opt, swap and insertion elevates the solution quality from $11.86 \%$ to $17.16 \%$ displaying the advantages. The effectiveness and stability of the proposed algorithm shed new light on the routing of MCV distribution problems in intelligent logistics.
\end{abstract}

(C) 2018 CPE, University of Maribor. All rights reserved.

\begin{tabular}{l} 
A R T I C L E I N F O \\
\hline Keywords: \\
Intelligent logistics; \\
Vehicle routing problem (VRP); \\
Multi-compartment vehicle (MCV); \\
Bionic optimization; \\
Fruit fly optimization algorithm \\
(FOA) \\
*Corresponding author: \\
3472181435@qq.com \\
(Li, S.W.) \\
Article history: \\
Received 3 September 2018 \\
Revised 4 December 2018 \\
Accepted 7 December 2018
\end{tabular}

\section{References}

[1] Dantzig, G.B., Ramser, J.H. (1959). The truck dispatching problem, Management Science, Vol. 6, No. 1, 80-91, $\underline{\text { doi: }}$ 10.1287/mnsc.6.1.80.

[2] Garey, M.R., Johnson, D.S. (1979). Computers and Intractability: A Guide to the Theory of NP-Completeness, W.H. Freeman \& Co., New York, USA.

[3] Pan, W.-T. (2012). A new fruit fly optimization algorithm: Taking the financial distress model as an example, Knowledge-Based Systems, Vol. 26, 69-74, doi: 10.1016/j.knosys.2011.07.001.

[4] Gylienè, V., Eidukynas, V. (2016). The numerical analysis of cutting forces in high feed face milling, assuming the milling tool geometry, Procedia CIRP, Vol. 46, 436-439, doi: 10.1016/j.procir.2016.03.132.

[5] Davim, J.P. (2011). Modern machining technology: A practical guide, Elsevier, Amsterdam, Netherlands, doi: $10.1533 / 9780857094940$.

[6] Ji, W., Liu, X., Wang, L., Sun, S. (2015). Experimental evaluation of polycrystalline diamond (PCD) tool geometries at high feed rate in milling of titanium alloy TC11, The International Journal of Advanced Manufacturing Technology, Vol. 77, No. 9-12, 1549-1555, doi: 10.1007/s00170-014-6517-9.

[7] Mitić, M., Vuković, N., Petrović, M., Miljković, Z. (2015). Chaotic fruit fly optimization algorithm, Knowledge-Based Systems, Vol. 89, 446-458, doi: 10.1016/i.knosys.2015.08.010.

[8] Han, J., Liu, C. (2013). Fruit fly optimization algorithm based on bacterial chemotaxis, Journal of Computer Application, Vol. 33, No. 4, 964-966, doi: 10.3724/SP.J.1087.2013.00964.

[9] Chajakis, E.D., Guignard, M. (2003). Scheduling deliveries in vehicles with multiple compartments, Journal of Global Optimization, Vol. 26, No. 1, 43-78, doi: 10.1023/A:1023067016014.

[10] Avella, P., Boccia, M., Sforza, A. (2004). Solving a fuel delivery problem by heuristic and exact approaches, European Journal of Operational Research, Vol. 152, No. 1, 170-179, doi: 10.1016/S0377-2217(02)00676-8. 
[11] El Fallahi, A., Prins, C., Calvo, R.W. (2008). A memetic algorithm and a tabu search for the multi-compartment vehicle routing problem, Computers \& Operations Research, Vol. 35, No. 5, 1725-1741, doi: 10.1016/i.cor.2006. 10.006 .

[12] Chen, P., Huang, H.-K., Dong, X.-Y. (2009). A multi-operator based iterated local search algorithm for the capacitated vehicle routing problem, Journal of Beijing Jiaotong University, Vol. 33, No. 2, 1-5.

[13] Muyldermans, L., Pang, G. (2010). On the benefits of co-collection: Experiments with a multi-compartment vehicle routing algorithm, European Journal of Operational Research, Vol. 206, No. 1, 93-103, doi: 10.1016/j.ejor.2010. $\underline{02.020}$.

[14] Reed, M., Yiannakou, A., Evering, R. (2014). An ant colony algorithm for the multi-compartment vehicle routing problem, Applied Soft Computing, Vol. 15, 169-176, doi: 10.1016/i.asoc.2013.10.017.

[15] Gajpal, Y., Abad, P. (2009). An ant colony system (ACS) for vehicle routing problem with simultaneous delivery and pickup, Computers \& Operations Research, Vol. 36, No. 12, 3215-3223, doi: 10.1016/i.cor.2009.02.017.

[16] Balseiro, S.R., Loiseau, I., Ramonet, J. (2011). An ant colony algorithm hybridized with insertion heuristics for the time dependent vehicle routing problem with time windows, Computers \& Operations Research, Vol. 38, No. 6, 954-966, doi: 10.1016/j.cor.2010.10.011.

[17] De la Cruz, J.J., Paternina-Arboleda, C.D., Cantillo, V., Montoya-Torres, J.R. (2013). A two-pheromone trail ant colony system - Tabu search approach for the heterogeneous vehicle routing problem with time windows and multiple products, Journal of Heuristics, Vol. 19, No. 2, 233-252, doi: 10.1007/s10732-011-9184-0.

[18] Valíček, J., Harničárová, M., Öchsner, A., Hutyrová, Z., Kušnerová, M., Tozan, H., Michenka, V., Šepelák, V., Mital', D., Zajac, J. (2017). Quantifying the mechanical properties of materials and the process of elastic-plastic deformation under external stress on material, Materials, Vol. 8, No. 11, 7401-7422, doi: 10.3390/ma8115385.

[19] Yousefikhoshbakht, M., Didehvar, F., Rahmati, F. (2014). Solving the heterogeneous fixed fleet open vehicle routing problem by a combined metaheuristic algorithm, International Journal of Production Research, Vol. 52, No. 9, 2565-2575, doi: 10.1080/00207543.2013.855337.

[20] Liu, Z.-X., Wang, Y.-F., Zhang, Y. (2014). Multiple population fruit fly optimization algorithm for automatic warehouse order picking operation scheduling problem, Journal of Wuhan University of Technology, Vol. 36, 71-77.

[21] Karpus', V.E., Ivanov, V.A. (2008). Universal-composite adjustable machine-tool attachments, Russian Engineering Research, Vol. 28, No. 11, 1077-1083, doi: 10.3103/S1068798X08110105.

[22] Zheng, X.-L., Wang, L., Wang, S.-Y. (2014). A hybrid discrete fruit fly optimization algorithm for solving permutation flow-shop scheduling problem, Control Theory \& Applications, Vol. 31, No. 2, 159-164.

[23] Lin, S. (1965). Computer solutions of the traveling salesman problem, The Bell System Technical Journal, Vol. 44, No. 10, 2245-2269, doi: 10.1002/j.1538-7305.1965.tb04146.x.

[24] Laporte, G. (1992). The vehicle routing problem: An overview of exact and approximate algorithms, European Journal of Operational Research, Vol. 59, No. 3, 345-358, doi: 10.1016/0377-2217(92)90192-C.

[25] Lin, S.-W., Lee, Z.-J., Ying, K.-C., Lee, C.-Y. (2009). Applying hybrid meta-heuristics for capacitated vehicle routing problem, Expert Systems with Applications, Vol. 36, No. 2, Part 1, 1505-1512, doi: 10.1016/j.eswa.2007.11.060.

[26] Larbi, A.A., Bounif, A., Bouzit, M. (2018). Comparisons of LPDF and MEPDF for lifted $\mathrm{H}_{2} / \mathrm{N}_{2}$ jet flame in a vitiated coflow, International Journal of Heat and Technology, Vol. 36, No. 1, 133-140, doi: 10.18280/ijht.360118.

[27] Ike, C.C. (2018). Exponential fourier integral transform method for stress analysis of boundary load on soil, Mathematical Modelling of Engineering Problems, Vol. 5, No. 1, 33-39, doi: 10.18280/mmep.050105.

[28] Abdulkader, M.M.S., Gajpal, Y., ElMekkawy, T.Y. (2015). Hybridized ant colony algorithm for the multi compartment vehicle routing problem, Applied Soft Computing, Vol. 37, 196-203, doi: 10.1016/i.asoc.2015.08.020. 


\section{APEM}

\title{
Optimizacija s hibridnim algoritmom mušic za reševanje usmerjanja večprostorskih vozil v inteligentni logistiki
}

\author{
Wang, C.L. , Li, S.W. ${ }^{\mathrm{a},{ }^{*}}$ \\ ${ }^{\mathrm{a}}$ School of Business, Shandong Normal University, Ji'nan, P.R. China
}

\begin{abstract}
POVZETEK
Namen študije je bil rešiti problem usmerjanja večprostorskih vozil v inteligentni logistiki s pomočjo optimizacije $\mathrm{z}$ algoritmom mušic (FOA). Za reševanje problema usmerjanja večprostorskih vozil (MCVRP) v inteligentni logistiki z uporabo optimizacije diskretnega prostora, je bil uporabljen hibridni FOA (HFOA) s tremi integriranimi lokalnimi iskalnimi metodami (2-opt, zamenjava, vstavljanje). Numerični eksperimenti potrdijo večjo zmogljivost algoritma HFOA, vključno z izboljšanjem celotne dolžine poti in kakovostjo rešitve, za vse predlagane probleme. Pri 50 strankah je ugotovljeno skrajšanje celotne dolžine poti 3,21 \%, pri 150 strankah pa 9,83 \%, kar kaže, da je ta HFOA učinkovitejši v večjem merilu. HFOA z integriranimi iskalnimi metodami izboljša kakovost rešitve iz 11,86 \% na 17,16 \%. Učinkovitost in stabilnost predlaganega algoritma prikažeta problem usmerjanja večprostorskih vozil v inteligentni logistiki v novi luči.
\end{abstract}

(C) 2018 CPE, University of Maribor. All rights reserved.

\section{PODATKI O ČLANKU}

Ključne besede:

Inteligentna logistika

Problem usmerjanja vozil

Večprostorsko vozilo

Bionična optimizacija

Optimizacija z algoritmom mušic

${ }^{*}$ Kontaktna oseba:

3472181435@qq.com

(Li, S.W.)

Zgodovina članka:

Prejet 3. septembra 2018

Popravljen 4. decembra 2018

Sprejet 7. decembra 2018 